\title{
Trivium
}

Revue franco-allemande de sciences humaines et sociales - Deutsch-französische Zeitschrift für Geistesund Sozialwissenschaften

$8 \mid 2011$

Traductions et transferts des savoirs dans l'espace euro-méditerranéen à l'époque médiévale

\section{Übersetzung aus dem Arabischen und lateinisches medizinisches Vokabular: einige Beispiele}

\section{Danielle Jacquart et Gérard Troupeau}

Traducteur : Andreas Pfeuffer

\section{OpenEdition}

\section{Journals}

Édition électronique

URL : http://journals.openedition.org/trivium/3877

DOI : 10.4000/trivium.3877

ISSN : 1963-1820

Éditeur

Les éditions de la Maison des sciences de l'Homme

\section{Référence électronique}

Danielle Jacquart und Gérard Troupeau, « Übersetzung aus dem Arabischen und lateinisches medizinisches Vokabular: einige Beispiele », Trivium [Online], 8 | 2011, online erschienen am 16 Mai 2011, abgerufen am 08 September 2020. URL : http://journals.openedition.org/trivium/3877 ; DOI https://doi.org/10.4000/trivium.3877

Ce document a été généré automatiquement le 8 septembre 2020

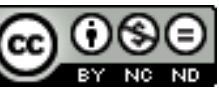

Les contenus des la revue Trivium sont mis à disposition selon les termes de la Licence Creative Commons Attribution - Pas d'Utilisation Commerciale - Pas de Modification 4.0 International. 


\title{
Übersetzung aus dem Arabischen und lateinisches medizinisches Vokabular: einige Beispiele
}

\author{
Danielle Jacquart et Gérard Troupeau \\ Traduction : Andreas Pfeuffer
}

\section{NOTE DE L'ÉDITEUR}

Wir danken Danielle Jacquart und den Éditions du Centre national de la recherche scientifique für die freundliche Genehmigung, diesen Artikel zu übersetzen.

1 Die in Italien und Spanien zwischen dem 11. und dem 13. Jahrhundert entstandenen lateinischen Übersetzungen von arabischen Werken eignen sich bestens für eine Untersuchung des mittelalterlichen medizinischen Vokabulars. Ihre Verfasser blieben zwar der vorher existierenden Terminologie verhaftet, sie leisteten jedoch einen Beitrag zur Etablierung einer neuen. Genau diesen Übergang von einer Terminologie zu einer anderen wollen wir veranschaulichen. Wir stützen uns dabei auf Texte, für die wir eine arabisch-lateinische Edition erstellt haben. ${ }^{1}$

Diese Übersetzungen sind für eine Untersuchung des Vokabulars vor allem deswegen von Interesse, weil sie zu unterschiedlichen Zeiten entstanden sind und sich an ihnen somit eine bestimmte Entwicklung nachzeichnen lässt. Die beiden ersten, die ohne Zweifel italienischen Ursprungs sind, wurden vor dem 12. Jahrhundert erstellt; die anderen, auf der Iberischen Halbinsel entstandenen Übersetzungen stammen aus der Periode zwischen dem 12. und dem Ende des 13. Jahrhunderts. 


\section{Herangezogene Übersetzungen, chronologisch geordnet ${ }^{2}$}

- Ysagoge Iohannitii: im Allgemeinen Constantinus Africanus (gest. 1087) zugeordnet; erste lateinische Version der Masā'il fi 'ț-țibb des Ḥunayn b. Isḥāq; sie berücksichtigt nur einen Teil des arabischen Textes.

- Aphorismi Iohannis Damasceni, anonyme Version der Nawādir aț-țibb des Yūhannā b. Māsawayh, sicherlich in Italien gegen Ende des 11. Jahrhunderts entstanden.

- Zehntes Buch des Liber ad Almansorem: Übersetzung des Kitāb al-Manșūrī von ar-Rāzī durch Gerhard von Cremona (1114-1187).

- Liber de secretis Rasis: in Santarém durch den Dominikaner Aegidius von Portugal (gest. 1265) erstellt"; ${ }^{3}$ es bietet nicht nur die Übersetzung der Maqāla fi sirr șina'at aț-țibb des ar-Rāzī, sondern auch eine weitere Version des Nawādir aț-țibb des Ibn Māsawayh.

- Questiones medicinales: zweite und vollständige Version der Masā'il fi 'țțibb des Hunayn b. Ishāā; einem gewissen Rufinus von Alexandria zugeschrieben ${ }^{4}$, der sich um 1270-1280 in Murcia aufhielt.

3 Das in den Werken der arabischen Gelehrten verwendete und somit übersetzte Vokabular kommt im Wesentlichen aus der Physiologie, der Pathologie und der Pharmakopöe. Außer den gängigsten Termini finden sich darin kaum anatomische Fachbegriffe. Anhand von einigen Beispielen soll zunächst auf die Entscheidungen eingegangen werden, die die verschiedenen Übersetzer bei der Übertragung von Begriffen aus der allgemeinen medizinischen Theorie getroffenen haben, bevor dann Gérard Troupeau eine Analyse des pharmazeutischen Vokabulars präsentieren wird.

\section{Einige Begriffe aus der Physiologie und Pathologie}

Die Physiologie der arabischen Ärzte und ihrer westlichen Kollegen des Mittelalters beruht auf der Vorstellung von /Komplexion` bzw. Konstitution und Temperament, die wiederum aus der Humoraltheorie herrührt, wie sie Hippokrates und Galen aufgestellt haben. Das von den lateinischen Autoren des 13. bis zum 15. Jahrhundert im Allgemeinen gebrauchte Wort zur Bezeichnung des Begriffs Temperament ist complexio, während das Wort temperamentum vor allem ein ausgeglichenes Temperament bezeichnet. So definiert Albertus Magnus den Begriff complexio als die sich aus der anteilsmäßigen Kombination der vier Grundeigenschaften Warm, Kalt, Feucht und Trocken ergebende Eigenschaft (proportionaliter in se habens omnes qualitates elementorum), während er den Ausdruck temperamentum complexionis zur Bezeichnung der Mischung vorbehält, in der jede Eigenschaft gleichermaßen vertreten ist. ${ }^{5}$ Die Wörter complexio und temperamentum werden in unseren Übersetzungen in unterschiedlicher Weise verwendet. Der dem griechischen кра̃ Konstitution, Temperament, entsprechende arabische Begriff lautet mizāğ; seine Übertragung ins Lateinische scheint den frühen Übersetzern einige Schwierigkeiten bereitet zu haben.

Die zwei in beneventinischer Schrift geschriebenen Manuskripte des 11. Jahrhunderts, in denen die ältesten Spuren der von Constantin übersetzten Isagoge ${ }^{6}$ erhalten sind, bieten in der Definition mit commixtio die wörtliche Übersetzung der Hauptbedeutung 
des Arabischen (aus einer Mischung herrührende Verbindung), aber nicht ihrer medizinischen Bedeutung; im Rest des Textes wird mizāğ meistens mit natura oder dessen Ableitungen naturalis, innaturalis und zwei Mal mit qualitas wiedergegeben. Die Handschriftenüberlieferung der Isagoge zeigt jedoch, dass das Wort commixtio rasch mit complexio glossiert ${ }^{7}$, ja sogar dadurch ersetzt wurde; dagegen wurden die Wörter natura und qualitas beibehalten, da im lateinischen Text nichts darauf hinwies, dass sie die spezielle Bedeutung von Komplexion/Konstitution ausdrückten.

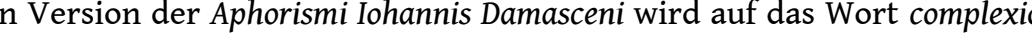
meist zurückgegriffen, um mizāğ wiederzugeben (12 Mal), man verspürt jedoch ein gewisses Schwanken; es finden sich auch natura, consonantia oder corpus. Des Weiteren gibt complexio zugleich das arabische imtizāğ wieder, das nur Mischung und nicht Temperament bedeutet, oder ta'liff, was Verbindung oder Zusammensetzung heißt.

7 In der Übersetzung des Liber ad Almasorem von Rhazes (ar-Rāzī) durch Gerhard von Cremona und in den Questiones medicinales Rufins findet sich konstant die Entsprechung mizāğ - complexio. Es scheint also, als habe sich mit dem 12. Jahrhundert die spezifisch medizinische Bedeutung von complexio durchgesetzt.

Die bei den frühen Übersetzern bereits festgestellte Schwierigkeit, eine lateinische Entsprechung für mizāğ zu finden, ist ein Anreiz, nach dem Vorkommen des Wortes complexio vor der Einführung arabisch-lateinischer Übersetzungen zu suchen. Die Wörterbücher liefern diesbezüglich nur wenige Informationen: Das 1976 erschienene Mittellateinische Wörterbuch führt keine der Schule von Salerno vorangehenden Beispiele auf ${ }^{8}$, während dem Thesaurus linguae latinae zufolge die klassische Bedeutung von complexio, nämlich Umfassung, Umarmung, Verknüpfung, schon zwischen dem 4. und dem 6. Jahrhundert bei einigen Autoren eine medizinische Färbung hatte. ${ }^{9}$ Eine Durchsicht der verbreitetsten medizinischen Handschriften des Hochmittelalters lässt auf den Nicht-Gebrauch von complexio schließen; der Begriff кра̃ бıৎ wurde tatsächlich anders zum Ausdruck gebracht.

Das in den vor-salernitanischen Handschriften häufig abgeschriebene De Medicina von Cassius Felix bietet folgende Äquivalenz: crasis id est natura vel temperantia. ${ }^{10}$ Auf das Wort temperantia stößt man auch im vierten Buch der Etymologiae des Isidor von Sevilla ${ }^{11}$; und in einem vor-salernitanischen Kommentar zu Galens De pulsibus ad Tirones $^{12}$ ebenso wie in der alten griechisch-lateinischen Version der Aphorismen des Hippokrates wird das Wort mit dem benachbarten Begriff temperies wiedergegeben. ${ }^{13}$

10 Wie schon gesehen, wurde die erste von Cassius Felix vorgeschlagene Äquivalenz, also natura und nicht temperantia, von den Übersetzern aus dem Arabischen bevorzugt gebraucht, bevor sich das Wort complexio durchsetzte. Bei unseren Übersetzern dienten die Ableitungen des Verbs temperare eher dazu, die spezielle Bedeutung von rausgeglichene Konstitution` wiederzugeben, die im Arabischen durch das Wort i'tidāl ausgedrückt wurde; während nämlich in der constantinischen Version der Isagoge dieser Begriff mit equalitas, der wörtlichen Übersetzung des Arabischen (Gleichgewicht) wiedergegeben wird, findet sich in den anderen Texten beständig die Entsprechung temperamentum (oder seltener temperantia) - i'tidāl.

11 Unterm Strich scheint es so, als sei das Wort complexio, das hin und wieder bei einigen spätlateinischen Autoren mit medizinischer Bedeutung belegt ist, von den italienischen Übersetzern gegen Ende des 11. und zu Beginn des 12. Jahrhunderts aufgegriffen worden, um mizāă wiederzugeben, die arabische Entsprechung des griechischen

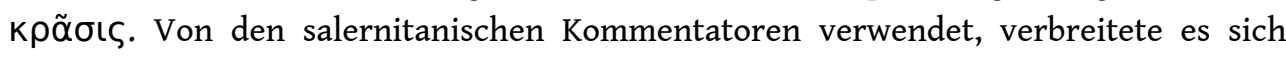


durch die Übersetzungen Gerhards von Cremona, vor allem der des Kitāb al-mizāğ, der arabischen Version des unter dem Titel De complexionibus bekannten Galenischen Traktats. ${ }^{14}$ Das Wort wurde das gesamte Mittelalter über zur Bezeichnung des allgemeinen Begriffs Temperament gebraucht, und dies umso mehr, als es ebenso in der von Burgundius von Pisa vorgenommenen griechisch-lateinischen Übersetzung des $\pi \varepsilon \rho \iota к \rho \alpha \sigma \varepsilon \tilde{\omega} \nu$ vertreten war. ${ }^{15}$

Eine neue Wendung nimmt das Ganze bei den Autoren der Renaissance. So wird in der 1543 in Basel unter dem Namen des Schweizerischen Gelehrten Alban Thorer gedruckten Paraphrase der Aphorismi Iohannis Damasceni das Wort complexio beständig durch die unterschiedslos verwendeten Begriffe temperamentum, temperantia und temperies ersetzt. Diese Lösung übernahm zu Beginn des 16. Jahrhunderts auch der neue Übersetzer des $\pi \varepsilon \rho \iota к \rho \alpha \sigma \varepsilon \tilde{\nu} \nu$, der Engländer Thomas Linacre. ${ }^{16}$ Mit dem 16. Jahrhundert kehrte man also wieder zur Terminologie zurück, wie sie vor den Übersetzungen aus dem Arabischen im Gebrauch gewesen war, und, als gäbe es kein lateinisches Wort mehr zur Bezeichnung einer ausgewogenen Zusammensetzung, griff man mit dem Adjektiv eucratos auf das Griechische zurück.

Nach diesen Ausführungen zur Übersetzung eines Grundbegriffes der mittelalterlichen Medizin sollen noch zwei andere Typen der Bildung von Fachtermini behandelt werden. Das erste Beispiel betrifft eine Krankheitsbezeichnung: Weil die Medizingeschichte dem Rhazes die erste Differenzierung der Symptome der Masern zuschreibt, ist es interessant zu sehen, wie die Übersetzer das von diesem Autor gebrauchte Wort al-ḥașba wiedergaben. Aegidius von Portugal gibt dieses Wort in seiner Version des De secretis als haceba wieder, obwohl er dessen Bedeutung verstanden zu haben scheint, da er es mit id est puncturas rubeas glossiert. Im 10. Buch des Liber ad Almansorem übersetzt Gerhard von Cremona es durch morbillus; das ist auch das Wort, mit dem er hașba im vierten Buch des Canon von Avicenna wiedergibt. Woher kommt dieses Wort morbillus, das im Lateinischen bis zum Ausgang des Mittelalters und selbst darüber hinaus noch verwendet wurde und das zum Altfranzösischen morbilles ${ }^{17}$ und zu dem im Italienischen immer noch gebräuchlichen morbillo geführt hat?

mittellateinischen Wörterbücher tragen kaum zur Erklärung bei. Du Cange behauptet, morbillus sei eine Verkleinerungsform von morbus (Krankheit). ${ }^{18}$ Das Novum Glossarium mediae latinitatis führt es erst gar nicht auf. Anscheinend wurde das Wort vor Gerhard von Cremona nicht verwendet, doch müsste man noch mehr Recherchen betreiben, um diese These zu untermauern. Falls es sich um einen vom spanischen Übersetzer geprägten Neologismus handelt, könnte man diesen durch Rückgriff auf die von Avicenna aufgestellte Definition der Masern erklären: Diesem Autor zufolge gibt es keinen Unterschied zwischen Pocken und Masern, abgesehen davon, dass die Letzteren weniger gravierend (minoris quantitatis, minor variolis) und weniger gut sichtbar sind (minus accidit oculo quam variole). ${ }^{19}$ Da Pocken und Masern bei Rhazes und Avicenna stets miteinander in Verbindung gebracht werden, würde sich die Wahl des Diminutivs durch den Vergleich zwischen den beiden Leiden erklären: Die Masern sind eine den Pocken ähnliche, aber weniger schwere Krankheit.

Das letztgenannte Beispiel für die Bildung eines Fachbegriffs bezieht sich auf die Eigenschaften des Pulses. In den Übersetzungen Rufins und Gerhards von Cremona findet sich der Ausdruck pondus pulsus, der dem arabischen wazn an-nabọ entspricht. Die erste und am weitesten verbreitete Bedeutung des arabischen wazn ist in der Tat 
Gewicht (wodurch sich das lateinische pondus erklärt), in der poetischen Fachsprache bezeichnet man damit allerdings das Versmaß. Da es in den betreffenden Passagen um den Rhythmus des Pulses geht, muss also die letztgenannte Bedeutung in Betracht gezogen werden. Es ist höchst erstaunlich, dass die Übersetzer diese spezifische Bedeutung des Wortes wazn nicht erfasst haben, da zum einen der Kontext offenkundig, zum andern der Vergleich mit musikalischen oder poetischen Rhythmen in der Medizin des 12. Jahrhunderts gängige Praxis war. In dem weit verbreiteten De pulsibus des Philaret beispielsweise ist die Rede von rithinon seu numerus pulsus. ${ }^{20}$ Aus jener Sinnentstellung, die in der Verwendung des Wortes pondus anstelle von numerus oder rythmus besteht, ging die Bildung eines von den mittelalterlichen Ärzten völlig eindeutig gebrauchten Terminus technicus hervor. So ist in dem im 14. Jahrhundert von Pietro d'Abano verfassten Conciliator in einer dem Pulsschlag gewidmeten Frage ${ }^{21}$ das Wort pondus zwölfmal ohne Erklärung bezeugt und nur ein einziges Mal nach der fünften Zitierung von dem Kommentar pondus vel rithimus begleitet. Die Verbreitung dieser Bedeutung des Wortes pondus wurde nämlich nicht nur durch die Übersetzung des Liber ad Almansorem gewährleistet, sondern auch durch die ebenfalls von Gerhard von Cremona angefertigte Übersetzung des Canon von Avicenna.

Die eben vorgestellten Beispiele verdeutlichen die unterschiedlichen Verfahren, die die mittelalterlichen Übersetzer zur Wiedergabe von Begriffen anwandten, für die sie kein lateinisches Äquivalent kannten, einmal abgesehen von simplen Transliterationen oder dem von Guy Beaujouan in seiner Darstellung ${ }^{22}$ erwähnten Rückgriff auf Bilderwörter. Der Fall der Wörter complexio und temperamentum bezeugt die Verwendung in einer eingeschränkteren und fachspezifischen Bedeutung von bekannten Begrifflichkeiten, die im klassischen Latein und in der Sprache des Hochmittelalters ein breiteres Bedeutungsspektrum hatten. Die Neuschöpfung morbillus scheint sich aufgrund der von den arabischen Autoren vorgeschlagenen Differentialdiagnostik zwischen Krankheiten angeboten zu haben. Was den Ausdruck pondus pulsus anbelangt, so fällt er in die so umfangreiche Kategorie von Sinnentstellungen, aus denen dann ein echter Terminus technicus hervorgegangen ist.

\section{Das Vokabular der Pharmakopöe}

17 Im Laufe unserer Untersuchung $\mathrm{zu}$ den lateinischen Übersetzungen arabischer Traktate, die Danielle Jacquart eben vorgestellt hat, haben wir eine Bestandsaufnahme des gesamten, in zweien dieser Traktate enthaltenen medizinischen Vokabulars vorgenommen, nämlich in dem zu Beginn des 13. Jahrhunderts von Aegidius von Portugal übersetzten Liber de Secretis von Rhazes und der gegen Ende des 13. Jahrhunderts von Rufin von Alexandria übersetzten Isagoge des Ḥunayn b. Isḥāq.

Auf diese Weise haben wir ein arabisch-lateinisches Wortverzeichnis von 274 Wörtern aus dem Bereich der Pharmakopöe erstellt, von denen 211 aus dem Liber de Secretis stammen und 63 aus der Isagoge. Im Vergleich zu anderen arabischen medizinischen Werken ist diese Zahl nicht sonderlich hoch, das den »einfachen" Medikamenten gewidmete Zweite Buch des Canon von Avicenna umfasst beispielsweise 754 solcher Wörter. Trotzdem erschien uns dieses Corpus ausreichend umfangreich, um eine mit Zahlen arbeitende Untersuchung des Vokabulars der arabisch-lateinischen Pharmakopöe in Angriff nehmen zu können, eine Untersuchung, die eine Anzahl lexikographischer Informationen geliefert hat, die ich hier kurz vorstellen möchte. 
1. Herstellung »zusammengesetzter" Medikamente: pflanzliche Substanzen, tierische Substanzen und mineralische Substanzen. In welchem Verhältnis stehen die Wörter, die diese drei Arten von Substanzen bezeichnen? In den beiden ausgewerteten Traktaten machen die zur Bezeichnung der pflanzlichen Substanzen verwendeten Wörter $80 \%$ des gesamten Wortverzeichnisses aus, während diejenigen für tierische Substanzen nur $14 \%$ und diejenigen für mineralische Substanzen nur $6 \%$ ausmachen. Das bedeutet, dass botanische Begriffe bei Weitem die größte Masse des Vokabulars der Pharmakopöe ausmachen, das die mittelalterlichen Übersetzer ins Lateinische $\mathrm{zu}$ übertragen hatten. Und genau das ist auch die Wortkategorie, die ihnen am meisten Schwierigkeiten bereitete, während die Übersetzung von tierischen und mineralischen Substanzen sie vor geringere Probleme stellte.

21 Andererseits ist das Vokabular der arabischen Pharmakopöe nicht homogen. Es setzt sich aus drei Sorten von Wörtern zusammen, nämlich arabischen, persischen und griechischen. In welchem Verhältnis stehen diese drei Arten von Wörtern zueinander? In den zwei ausgewerteten Traktaten stellen die Wörter arabischer Herkunft $68 \%$ des Lexikons dar, während die Wörter persischer oder griechischer Herkunft nur 21 \% bzw. $10 \%$ des Gesamtvokabulars ausmachen. Nun musste die relativ hohe Anzahl von persischen ${ }^{23}$ Begriffen, für die die Araber selbst kein entsprechendes arabisches Wort haben finden können, die lateinischen Übersetzer des Mittelalters vor ernste Probleme stellen, da sie schlicht kein Persisch konnten und ganz sicher nicht über einen Wortschatz, erst recht nicht über Informanten für diese Sprache verfügten. Wörtern zusammen: lateinischen Wörtern, latinisierten griechischen Wörtern und transliterierten Wörtern. In welchem Verhältnis stehen nun diese drei Arten von Wörtern zueinander? In den beiden ausgewerteten Traktaten machen die lateinischen Wörter nur 50 \% des Vokabulars aus, während die Wörter griechischer Herkunft 30 \%, die transliterierten $20 \%$ ausmachen. Auffallend an diesem Wortverzeichnis ist die im Vergleich zum arabischen Vokabular weitaus höhere Anzahl an Wörtern griechischer Herkunft und die Anzahl der transliterierten Wörter. Es besteht kein Zweifel daran, dass diese hohe Zahl von Fremdwörtern, die die Hälfte des Vokabulars der Pharmakopöe ausmachen, den lateinischen Übersetzungen ihren oft barbarisch anmutenden Charakter verleiht.

2.

Wir können aus diesem Wortverzeichnis zweitens noch eine Reihe von Informationen zu den von den mittelalterlichen Übersetzern angewandten Verfahren bei der Übertragung der arabischen, persischen und griechischen Wörter ins Lateinische ableiten.

24 a) Das erste Verfahren besteht in der Übersetzung durch ein lateinisches Wort. Diesbezüglich zeigt das Wortverzeichnis, dass $53 \%$ der arabischen, $22 \%$ der persischen 
und $9 \%$ der griechischen Wörter durch ein lateinisches Wort übersetzt wurden. Das heißt, dass die Übersetzer nur für die Hälfte der arabischen Wörter eine lateinische Entsprechung haben finden können und dass sie für eine gewisse Anzahl von persischen und griechischen Wörtern eine Entsprechung fanden, während die Araber keine dafür gefunden hatten. ${ }^{24}$

b) Das zweite angewandte Verfahren ist die Übersetzung durch ein Wort griechischer Herkunft. Es sind natürlich die griechischen Wörter, die nach dieser Vorgehensweise wiedergegeben wurden. Doch wenn das Wortverzeichnis zeigt, dass $70 \%$ der griechischen Wörter durch dasselbe Wort wiedergegeben wurden, zeigt es auch, dass $15 \%$ davon durch ein anderes griechisches Wort und $9 \%$ durch ein lateinisches Wort wiedergegeben wurden. ${ }^{25}$ Zum andern zeigt das Wortverzeichnis, dass $26 \%$ der persischen und $22 \%$ der arabischen Wörter durch ein latinisiertes griechisches Wort übersetzt wurden. ${ }^{26}$

c) Das dritte Verfahren ist die Transliteration. Wenn die Übersetzer des Mittelalters kein entsprechendes lateinisches oder griechisches Wort finden konnten, transliterierten sie das Wort, ob es nun arabischen oder persischen Ursprungs war. Doch das Wortverzeichnis zeigt, dass es vor allem persische Wörter waren, die transliteriert wurden, nämlich $42 \%$ gegenüber nur $17 \%$ der arabischen Wörter. Manchmal haben die Kopisten Glossen zur Erläuterung der Bedeutung des transliterierten Wortes eingefügt. ${ }^{27}$ Übrigens sind die transliterierten Wörter besonders interessant für die französische Lexikographie, da eine ganze Reihe dieser Wörter Eingang ins französische Vokabular der Pharmakopöe gefunden haben. ${ }^{28}$ Doch wurden die transliterierten Wörter von den Schreibern, dann von den Editoren der Renaissance derart entstellt, dass sie häufig nicht wiederzuerkennen sind, und genau aus diesem Grund ist es so schwer, das von den Übersetzern angewandte Transliterationssystem zu rekonstruieren.

d) Das vierte Verfahren ist die Übersetzung durch ein aus einer europäischen Vernakularsprache entlehntes Wort. In den beiden ausgewerteten Traktaten haben wir dieses Verfahren nur für die Übersetzung von zwei Worten finden können: mušmuš (»Aprikose«); übersetzt mit dem spanischen albaricóque, und badinğān (»Aubergine«), übersetzt mit dem spanischen berenjéna.

3.

Wir können aus diesem Wortverzeichnis drittens Informationen hinsichtlich des Grades an Ähnlichkeit oder Unähnlichkeit des Vokabulars der verschiedenen Übersetzer ableiten.

29 Aus dem Vergleich des Vokabulars des Liber de Secretis und desjenigen der Isagoge schließen wir, dass von den 26 Wörtern, die ihnen gemeinsam sind, Aegidius von Portugal und Rufin von Alexandria 25 mit demselben lateinischen Wort übersetzt haben und nur ein einziges mit zwei unterschiedlichen Wörtern. Das bedeutet, dass die beiden im 13. Jahrhundert wirkenden Übersetzer - der eine im portugiesischen Santarém, der andere im spanischen Murcia - beinahe die gleiche Terminologie verwendeten.

Vergleichen wir aber das Vokabular des Liber de Secretis und das der Isagoge mit dem des Canon von Avicenna, Vokabulare, denen 160 bzw. 56 Wörter gemeinsam sind, so stellen wir fest, dass Aegidius von Portugal und Gerhard von Cremona 74 \% der Wörter mit 
demselben lateinischen Wort und $26 \%$ mit verschiedenen Wörtern übersetzt haben, während Rufin von Alexandria und Gerhard von Cremona $84 \%$ der Wörter durch dasselbe lateinische Wort und $16 \%$ durch unterschiedliche Wörter wiedergegeben haben.

Dies besagt, dass das Vokabular der Übersetzer des 13. Jahrhunderts nicht mehr exakt dem der Übersetzer des 12. Jahrhunderts entspricht; des Weiteren, dass der Grad an Ähnlichkeit mit den Übersetzern variiert, wobei das Vokabular Rufins von Alexandria dem Gerhards von Cremona näher ist als dem des Aegidius von Portugal.

Soweit die ersten Ergebnisse lexikographischer Untersuchungen, die uns auf dem Gebiet der arabisch-lateinischen Pharmakopöe des Mittelalters die Erstellung eines arabisch-lateinischen Wortverzeichnisses liefern kann, eines Verzeichnisses, das man durch die Auswertung weiterer arabischer Texte sowie weiterer lateinischer Übersetzungen fortführen müsste.

\section{BIBLIOGRAPHIE}

\section{Quellen}

Albertus Magnus, De vegetabilibus libri VII, Ed. E. Meyer / C. Jessen, Berlin 1867.

Cassius Felix, Cassii Felix De medicina, Ed. V. Rose, Leipzig 1879 (Bibliotheca scriptorum Graecorum et Romanorum Teubneriana).

Durling, R. (Hg.), Galenus latinus I, Burgundio of Pisa's translation of Galen's $\pi \varepsilon \rho \iota k \rho \alpha \sigma \varepsilon \tilde{\omega} \nu » D e$ complexionibus«, Berlin / New York 1976 (Ars medica, II, 6, 1).

Isidor von Sevilla, Etymologiae sive origines Isidori Hispalensis Episcopi Etymologiarvm sive Originvm libri XX, 2 Bde., Ed. W. M. Lindsay, Oxford u. a. 1957.

Kaeppeli O. P., T., Scriptores Ordinis Praedicatorum Medii Aevi, Bd. I, Rom 1970.

Kühn, C.G. (Hg.), Claudii Galeni Opera omnia, Bd. 1, Hildesheim 1964 (Wiederabdruck der Ausgabe Leipzig 1821).

Yūhannā ibn Māsawayh (Jean Mésué), Le livre des axiomes médicaux (»Aphorismi«). Édition du texte arabe et des versions latines, avec traduction française et lexique, par D. Jacquart und G. Troupeau, Genf 1980.

\section{Sekundärliteratur}

Beaujouan, G. (1981): „Le vocabulaire scientifique du latin médiéval «, in: La lexicographie du Latin médiéval et ses rapports avec les recherches actuelles sur la civilisation du Moyen Âge. Colloque 
international du Centre National de la Recherche scientifique, Paris, 18-21 octobre 1978, Paris, S. 345-354.

Beccaria, A. (1971): „Sulle trace di un antico canone latino di Ippocrate e di Galeno«, Italia mediovale e umanistica, 14, S. 1-23.

Du Cange, Ch. Du Fresne (1840-1850): Glossarium medice at infimce latinitatis, Paris.

Bayerische Akademie der Wissenschaften und Berlin-Brandenburgische Akademie der Wissenschaften (1976): Mittellateinisches Wörterbuch bis zum ausgehenden 13. Jahrhundert, Bd. II, Lfg. 17, München.

Sezgin, F. (1970): Geschichte des Arabischen Schrifttums, Band III: Medizin, Pharmazie, Zoologie, Tierheilkunde - bis ca. $430 \mathrm{H}$, Leiden.

Thorndike, L. / Kibre, P. (1963): Catalogue of Incipits of Medieval Scientific Writings in Latin, Cambridge (Mass.).

Wartburg, W. von (1969): Französisches Etymologisches Wörterbuch, Bd. 6, III, Basel.

Wickersheimer, E. (1966): Manuscrits latins de médecine du Haut Moyen Age dans les bibliothèques de France, Paris.

\section{NOTES}

1. Eine dieser Editionen ist erschienen: Yūhannā ibn Māsawayh, Le livre des axiomes médicaux.

2. Vgl. zu den lateinischen Übersetzungen Thorndike / Kibre (1963), Sp. 856, 824, 272, 248, 716; zu den arabischen Texten Sezgin (1970), S. 249 f., 233, 281 f., 286.

3. Aegidius von Portugal war vor seinem Eintritt in den Orden des Heiligen Dominikus einige Zeit lang an der Universität von Paris tätig; vgl. Kaeppeli, Scriptores Ordinis Praedicatorum Medii Aevi, S. $15 \mathrm{f}$.

4. Rufinus ließ sich durch seinen Herrn Dominicus Marrochinus helfen; zu Letzterem vgl. Kaeppeli, op. cit., S. $328 \mathrm{f}$.

5. Albertus Magnus, De vegetabilibus, Buch I, tr. II, Kap. VIII und Buch IV, tr. II, Kap. II.

6. Mss. Monte Cassino 225; Paris, Bibl. nat., nv. acq. lat. 1628. Diese Manuskripte sind die ältesten Zeugen einer arabisch-lateinischen Übersetzungstradition medizinischer Texte; vgl. Wickersheimer (1966), S. 145 f.

7. Seit Beginn des 12. Jahrhunderts in der Handschrift von Perugia, Bibl. Aug., N 90.

8. Im Mittellateinischen Wörterbuch (Bayerische Akademie der Wissenschaften und BerlinBrandenburgische Akademie der Wissenschaften [1976], Sp. 1081) werden unter den medizinischen Autoren Constantinus Africanus (De gradibus), Albertus Magnus, Maurus von Salerno (De urinis) zitiert.

9. Nämlich bei Firmicus Maternus, Cassiodor, Pseudo-Apuleius und dem Verfasser einer pseudoaugustinischen Predigt.

10. Kapitel 73, Ad elefantiasim; Cassii Felicis De medicina, Ed. Rose, S. 177.

11. IV , 5, 1 "Sanitas est integritas corporis, et temperantia nature ex calido et humido« (Ed. Lindsay).

12. »... quia commemoravit ibi de cor vel eius temperantias«, vgl. Beccaria (1971), S. 3.

13. Liber aphorismorum, VI, 37: "qui densas et frigidas matrices habent ... semen que in ex utrisque temperiem habent...« (Ms. Paris, Bibl. nat., lat. 7027, IX ${ }^{\mathrm{e}}$ siècle, f. 162 v).

14. Vgl. Thorndike / Kibre (1963), Sp. 1538. 
15. In der Übersetzung des Burgundius wird кра̃ ausgeglichene Temperament ( $\varepsilon \cup ̉ \kappa \rho \alpha \sigma \iota \alpha)$ wird mit eucrasia oder contemperantia wiedergegeben. Vgl. Durling, Galenus latinus I, S. 152 und 157.

16. Die Übersetzung des Thomas Linacre ist ediert in Kühn, Claudii Galeni Opera omnia, Bd.1, S. 509-694.

17. Das altfranzösische morbilles kommt entweder direkt aus dem Lateinischen oder indirekt über das Italienische; vgl. Wartburg (1969), S. 125.

18. Du Cange zitiert ein nach dem Werk Alexander von Tralles erstelltes Glossar: »turpedo... quidam morbillum, id est minimum morbum, dixerunt ...« (Bd. 4, S. 545).

19. "Scias quod omnis morbillus est variola cholerica. Et non est differentia inter ea ambo in plurimo reliquarum dispositionum, nisi quod morbillus est cholericus et quod ipse est minoris quantitatis et quasi ipse non pertransit cutem. Et morbillus est minor variolis et minus accidit oculo quam variole« (Ed. Mailand, 1473, Buch IV, fen 1, tr. 4, Kap. VII).

20. Vgl. Ms. Paris, Bibl. nat., lat. 7102, XIII ${ }^{\mathrm{e}}$ siècle, f. 204.

21. Ed. Venedig, 1521,Differentia 82.

22. Es handelt sich wohl um den im selben Tagungsband wie der vorliegende Artikel erschienenen Beitrag von Guy Beaujouan (1981); A.d.Ü.

23. Im Original offensichtlich irrtümlich »arabisch«; A.d.Ü.

24. Beispiele für mit einem lateinischen Wort übersetzte persische Wörter: Šāhtaranğ "Erdrauch«, übersetzt mit fumus terre; zinǧar »Grünspan«, übersetzt mit viridis grecus.

25. Beispiele für griechische Wörter, die durch ein anderes griechisches Wort wiedergegeben wurden: iqlimya »Ofenbruch« (vom griechischen kadmia); wiedergegeben mit scoria (vom griechischen skôria); qirtas »Papier« (vom griechischen khartôs), wiedergegeben mit papyrus (vom griechischen papuros); Beispiele für griechische Wörter, die durch ein lateinisches Wort wiedergegeben wurden: bunduq »Haselnuss« (vom griechischen pontikos), übersetzt mit avellana; lubya (vom griechischen lobos), übersetzt mit faseolus.

26. Bezüglich der latinisierten griechischen Wörter sei darauf hingewiesen, dass in das Wörterbuch von Du Cange einige von ihnen aufgenommen wurden. $\mathrm{Zu}$ dem Wort spodium (vom griechischen spodion), das die Knoten des Bambusrohrs bezeichnet, sagt er: Spodium est Res cujus origo nos latet, res tamen adusta; videlicet Avicenna dicit radices cannarum adustarum.

27. Hier einige Beispiele für diese Glossen $\mathrm{zu}$ transliterierten Wörtern: darsesahan (»Aspalathholz/Aspalathus«): id est arbor malorum granatorum silvestrium; sandaraca (»Sandarach«): id est gumma quod mastico assimilatur et ex eo fit vernix; kanebir (»Lerchen«): id est columbe silvestres que in domibus non nutriantur.

28. Als Beispiele lassen sich französische Wörter persischen Ursprungs anführen: emblique (Emblika), gingembre (Ingwer), lazuli (Lapislazuli), laque (Lack), nénuphar (Seerose); arabischen Ursprungs sind: camphre (Kampfer), caroube (Johannisbrot, Karube), santal (Sandelholzbaum), séné (Sennesstrauch), sumac (Sumach), tamarin (Tamarindenfrucht).

\section{INDEX}

Schlüsselwörter : lateinische Übersetzungen arabischer Traktate, medizinisches Vokabular, Pharmakopöe

Mots-clés : traductions latines de traités arabes, vocabulaire médical, pharmacopée 


\section{AUTEURS}

\section{DANIELLE JACQUART}

Danielle Jacquart ist Professorin an der Ecole Pratique des Hautes Etudes (EPHE). Nähere Informationen finden Sie hier.

\section{GÉRARD TROUPEAU}

Gérard Troupeau (1927-2010) war Professor am Institut national des langues et civilisations orientales (INALCO) und an der Ecole Pratique des Hautes Etudes (EPHE). Nähere Informationen finden Sie hier. 